\title{
GIS-Anwendungen im Internet
}

\author{
Jens Fitzke
}

Ryerson University

\section{Claus Rinner}

Ryerson University

\section{Dirk Schmidt}

Ryerson University

Fitzke, J., Rinner, C., \& Schmidt, D. (1997). GIS-anwendungen im internet [GIS applications on the internet]. Geo-Informations-Systeme 6(97), 25-31.

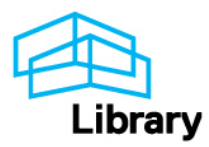




\title{
GIS-Anwendungen im Internet
}

\author{
Jens Fitzke, Claus Rinner und Dirk Schmidt, Bonn
}

\section{Zusammenfassung}

Der vorfiegende Beitrag gibt einen Uborblick über GIS-Anwendungen im internet. Nach einem praxisbezogenert Einstieg in die Thematik wird der technische Hintergrund von internet-GIS be: leuchtel. Der Betrachtung liegen drei derzeit identifizierbare Ausgangspositionen für derartige Implementationen zugrunde: die Browser-, die GIS- und die Komponenter-Perspektive. Angesichts des heterogenen Feides. Internot-GiS' Wird anschliebend eine konsistente Terminoiogie zur Klassifizierung von Internet-GiS.Anwendungen nach dem Spektrum ihrer 'Dienst'-Leistungen vorgeschiagen. Es wird gezeigt, wie diese einzein voneinander trennbaren Dienste ais funktionale Baustoino der verschiedenen Anwondungstypen aufgefaßt werden konnen Dieses Konzept kann als Basis for die künitige fachiche Diskussion dienen Abschließend wird in einem Fazit auf die absehbare lechnische Entwickiung und ihre möglichen Konsequenzen tur die Arbert von GiSExperten und GiS-Anwendern sowie für dert GIS Markt auf dem Weg ins nachste Jahrtausend eingegangen.

\section{Abstract}

This paper gives an overview ot GiS applications on the Internet starting with an introductory scenario. The technical background of Internet GIS is axamined from three perspectives: the browsercenterad view, the internef-enabled GIS yiew, and the component viow. Subse. quently, a consistent terminology for the classification of internet GIS applications, according to the services they oller, is proposed as a base for further discussion. The individual service components can be interpreted as functio. nal modules of different application types. The paper concludes with outlines of future technical aovances and possible resulting consecuences for GIS experts, Users and the GIS markot on the way into the next millenium.

\section{Einleitung}

Schlagworte wie "NetG/S:, Public Access G/S ${ }^{+}$oder "Communicating Geographically on the Web" sind derzeit auf GIS-Konferenzen und -Messen, in Zeitschifften und vor allem in den Werbebroschüren fast aller GISHersteller zu finden

Autgrund des interesses, das dem Internel in der öffentlichen Diskussion zukammt, werden auch bisherige Nicht-GIS-Anwender auf dieses neve Marktsegment aufmerksam. Dabei ist die Idee, Geographische informationen digital uber Netze zur Vertügung zu stellen, nicht neu. Bereits vor zwel Jahren war zu lesen, daß die digitale Karte (im internet) bald als Intormationsquelle genauso selostverständlich wie das Telefon genutzt werden wird: "Soon, interactive electronic maps will be as common an information tool as the telephone". (THOEN 1995). Auch wenn diese Vision in dem dargestellten Ausmal3 bis heute noch nicht Realitat geworden ist, so lajt sich doch gerade in jüngster Zeit ein starker Trend verschiedener GIS-Anwendungsbereiche in Richtung Internet feststellen.

En großes Problem bei der Auseinandersetzung mit dem Themenbereich Gis-Anwendungen im Internet' besteht in der Heterogenitát der momentan existierenden Anwendungsbelspiele, die sich hautig noch in einem prototypischen Zustand befinden. Bei den Entwicklungen, die tellweise sehr unterschiedliche Anwendungsziele verfalgen, wurde oft mit sehr viel Kreativitat das breite Feld technischer Müglichkeiten ausgeschopft. Die so entstandene Vielfalt technisch unterschiedlicher, von der Funktionalitat teilweise ahnlicher Realisierungen ist unter anderem aut das Fehlen vertug. barer kommerzieller Produkte zurückzufuhren. Naturgemaß weisen die verschiedenen Anwendungen Stärken und Schwachen in unterschiedichen Bereichen auf. Eine Bewertung karn daher immer nur vor derr Hintergrund konkreter Anforderungen erfolgen

Wie es schon so hăufig im Bereich GIS der Fall war, scheinen die in der Praxis entwickelten Prototypen nicht nü den GIS-Sotware-Anbietern, sondern auch der theoretischen Diskussion die Richtung vorzugeben f, the internst is changing how GIS is done: MATUSC-AK 1996), Inkonsistente Terminologie und fehlende Gliederungskonzepte machen es schwierig, bestehende WebGIS-Losungen einzuschatzen und eigene Anforderungen für diesen Bereloh zu formulieren. Daruber hinaus herrscht haufig Unklarheit daruber, was bereits implementierte Anwendungen oder kommerziell angebotene Soltware in der Lage sind zu leisten oder weiche GIS- ader InternetFunktionalitsten von ihnen unterstützt. werden.

\section{Szenario}

1. HerriFrau $X$ ist Okologe/OKologin und befaBt sich derzet mit der Biotopkartierung in A-Stadt. Dazu benutzt $X$ das Geographische intormationssystem $G$. $\times$ hat drei alternative Kartenentwürfe im Format des G-Systems erzeugt und will sie einigen Kollegen in anderen Instituten übermittein. X kopiert die Geodaten aut einen uber den WWWServer zuganglichen Bereich selnes Rechners und ersteilt eine einfache WWW-Seite, uber die seine Kollegen Intormationen zu den drei Karten erhalten und sie zum Download auswanlen kơnnen. Beim entsprectienden Klicken erhält der Kollege die Datei im G-Format und kann sie mit seinem lakalen $G$ ansehen.

2. Da $X$ seine Karten auch einem befreundeten Ingenieurbüro I in BDorf zugånglich machen wili, das jedoch kein G-System hat, erzeugt er die Karten ais Graphikdateien und erweitert seine WWW-Seite um entsprechende Auswahimöglichkeiten, $\mathrm{Da} X$ ein Standard-Rastertormat gewáhit hat, reicht dem ingenieurbüro ein gängiger WWWBrowser, um die Karten anzusshen

3. Es taucht jedoch der Wunson auf, in Verbindung mit der angezeigten Karte auch Auskuntt uber die Artenzusammensetzung der Giotope 
erhaiten zu können. X macht daher die Kartenprasentation in dem Sinne interaktiv, daß die von einem Benutzer auf der Kartengraphik im WWW-Browser angeklickten Koordinaten an seinen eigenen Rechner übermittelt werden. Dort wird ein Prozeß engestoßen, der sein GIS G startet, feststellt, welches Biotop ggt angeklickt wurde und die zugehorigen Sachdaten aus der Datenbank abfragt. Diese Tabelle wird exportiert und über die WWWVerbindung auf den Bildschirm des Benutzers ubertragen.

4. Als der Ingenieur I feststellt, daB die Geodaten und die Funktionen des G-Systems von X genau die Bedingungen erfüllen, die er fur ein anderes Projekt benötigt, tragt er beiX um einen Zugang zu dessen G-System an, da er sich für ein einzelnes Projekt kein komplettes GIS anschaffen möchte. Nach Kuărung der Rahmenbedingungen Bthet $X$ dem Ingenieur einen Online-Zugang auf die volle Funktionalităt seines GIS und auf die Bereiche der Datenbank, die die gewünschten Biotop-Daten enthaten. I schreibt sich ein Skript, mit dem er die ihn interessierenden Informationen aus X's Daten heraushoit.

5. Dann erhalt I von einem Kunden andere, gleichartige Daten im G-Format. Xermoglicht I ais zusatzliche DienstIeistung, daß I die fremden Daten auf seinen Rechner schicken und sein früher geschriebenes Skript daraut zur Ausfohrung bringt.

\section{Technologie-Bausteine}

Die Entwicklung von GIS-Anwendungen im Internet wird derzeit mit unterschiedlicher intensităt aus drel verschiedenen Ausgangspositionen heraus betrieben. Als Basis der bisher implementierten GIS-Anwendungen im Internet dient der WWW-Browser, dem zusatzilich Făhigkeiten erschlossen werden, um mit raumbezogenen Daten umzugehen. So wird aus Software zur Datenubertragung (connectivity) solche zur Datenverarbeitung (computing) (ERNST 1996). Umgekehrt konnen aber auch Datenverarbeitungsprogramme - in diesem Fall GIS - um Kommunikationsfunktionen etweitert werden, Die dritte Perspektive kann ais weitgehend voraussetzungsios charakterisiert werden, da sie auf neuen Techniken aufbaut, die von vornherein die Entwicklung integrativer Losungen erlauben.

In diesem Spannungsfeid zwischen Browser-basiectem Internet-GIS, Internet-fähigem GIS und objektorientierten GIS-Komponenten steht eine Reihe von Sottware-Techniken zur Verfugung, die letztendich die Entwicklungslinien zusammenführen werden. Um welche Techniken es sich dabei handelt und wie sie eingesetzt werden konnen, soll in den folgenden Abschnitten schlaglichtartig dargestelit werden (s. auch Fig. 2.2)

\section{1 connectivity first Browser-basiertes Internet-GIS}

Es wird von folgender Ausgangssituation ausgegangen: Der Benutzer verfagt uber einen Internet-AnschluB und die notwendige Kommunikationssoftware, einen WWW-Browser. Zusatzlich, aber nicht zwingend erforderlich, kann ein Geographisches Informationssystem lokal installiert sein. Im einfachsten Fall kann nun der Standard-Browser dazu benutzt werden, im Internet nach GIS-Daten zu suchen und diese - beispielsweise unter Verwendung des file transter protocol (FTP) - lokal zu speichern. Diese Daten kónnen

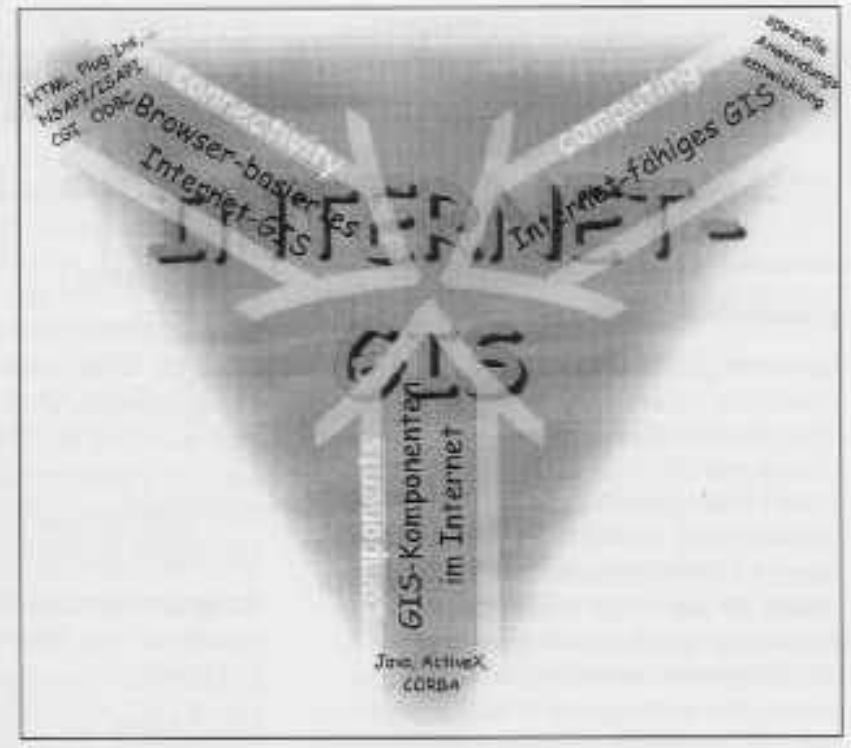

Fig. 2.7: Ausgangspositionen bei der Entwiokiung von GIS. Anwendungen im Internot

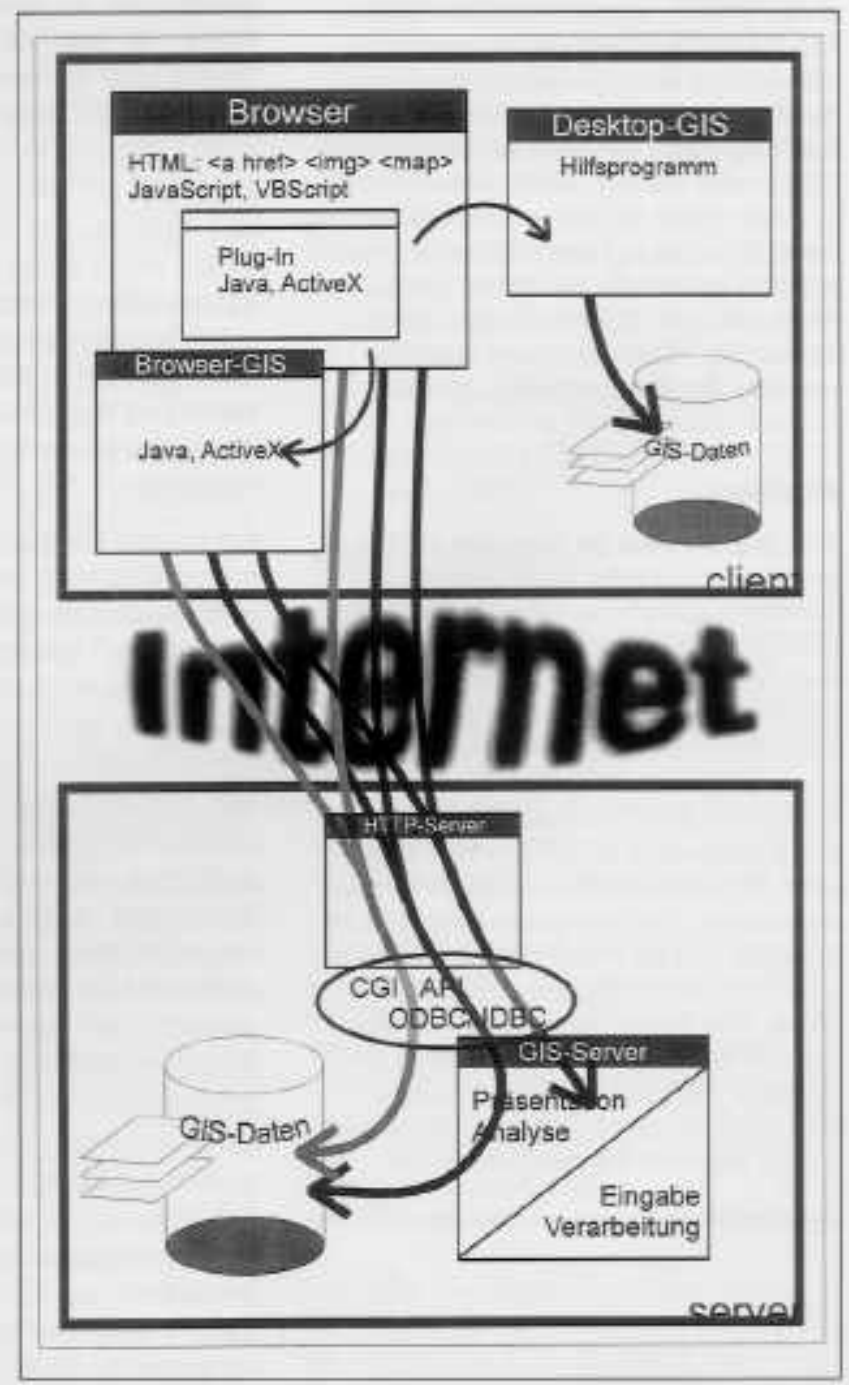

Fig. 2.2: Realisierung des internet-Zugriffs auf GIS-Ressourcen 
dann, unabhangig(!) von der InternetAnbindung, mit dem lokalen GIS verarbeitet werden. Von Integration kann allerdings erst gesprochen werden. wenn GIS-Sotware Internetprotokolle unterstutzt oder Kommunikationssoftware GIS-Erweiterungen erhait.

\subsubsection{Schianke Clients: GIS-Emulation im Browser}

Naheliegend und dem groberen $\mathrm{Be}$ nutzerpotential entsprechend beginnt die Entwicklung auf der Seite des Client, inderm die Benutzeroberflache des GIS im Browser nachgeahmt wird. Die GIS-Funktionen und -Daten werden auf dem Server zur Verfügung gestellt (s ehe 2.1.2).

\section{Schritt: Standard-HTML}

Zunáchst wird lediglich der Sprachumfang der Hypertext Markup Language (HTML) genutzt, um eine GIS-Benutzeraberlláche in Web-Seiten abzubilden, die von jedem graphikfahigen Browser angezeigt werden konnen. Als Minimaliosung umfaßt dies:

- Hyperlinks mit der HTML-Markie. rung <a href> und Steuerelemente wie Textfelder. Auswahllisten und Sçhalttlächen mit HTML-Formularen (<form) sowie

- Graphiktenster" mit <img> und $<m a p>$ (for client-side imagemap) bzw, <ismap> (server-side imagemap)

Mit diesen Hilfsmitteln laßt sich die Benutzeroberfläche eines vollstandigen GIS emulieren. Nachteil dieses Arsatzes ist nach derzeitigem Stand (HTML 3.2) die Beschrankung des Graphikfensters auf Rasterdaten (GIF, JPEG), die noch dazu nicht georeferenziert sind. Eine Georeferenzierung kann aber ubber die Bildkoordinaten und entsprechende Verarbeitungsroutinen auf dem Server vorgenommen werden. Neben diesen unzulanglichen (geo) graphischen Fahigkeiten besteht der wesentliche Nachtel dieser Losung darin, dab jede Benutzer-Aktion eine Antrage an den Server bodeutet und somit die Netzbelastung erhöht. Um von hier aus einen Schritt weiter zu gehen, können 1.) Teile der Steuermechanismen lokal auf dem Client ablauten und 2) intelligentere Graphikformate benutzt werden. Dies erläutern die folgenden zwei Abschnitte

\section{Schritt}

Lokale Steverung dunch Skriptsprachen

Die Skriptsprachen JavaScript (Netscape) und VBScript (Microsott), die direkt in HTML-Dokumente eingebettet sind und vom Browser interpretient werden, können dazu verwendet werden, einen Teil der Steuermechanismen lokal auszulchren. Dazu gehoren beispielsweise die Auswertung von Einträgen in Formularfeldern (Prüten auf Plausibilitat) oder einfache Berechnungen.

\section{Schritt: \\ Intelligente Graphik durch \\ Hilfsprogramme und Plug-Ins}

Von einem Geographischen informationssystem kann erst gesprochen werden, wenn mehr als nur Rasterbilder verarbeitet werden. Dies ist unter Verwendung von reinem HTML nur indirekt moglich, u. U. aber ausdrucklich erwünscht, da im Internet auf diese Weise nur ein - für die Weiterverarbeitung unbrauchbares - Abbild des Datenbestandes verfügbar ist.

Erkennt der Browser ein von ihm nicht direkt interpretierbares Datenformat (z.B. DXF, CGM, SHP, CGO USw.), so kann er diese Dalen an ein lokal installiertes Anwendungsprogramm weitergeben. Voraussetzung datur ist allerdings, daß der Benutzer uber der MIME-Typ diese Verknúptung zwischen Dateinamenerweiterung und Sottware selbst hergestellt hat. Wird die Anwendung als Hilfsprogramm gestartet, endet an dieser Stelle die Verbindung zwischen GIS und Interne: und es kann nicht mehr von GIS-Internet-Kopplung 1. e. S. gesprochen werden. Anders bem Plug-In: Hier wird das Anwendungsprogramm im Browser selbst ausgefuhrt, und die Möglichkeit der Kommunikation mit dem Server bleibl bestehen. Die derzeit unterstützlen Vektordaten-Formate DXF CGM und VRML entstammen allerdings eher dem Graphik-Bereich. ubertragen daher keine GIS-spezifischen Datenstrukturen

\subsubsection{Die andere Seite der Medallie. server-side processing}

Die Auslagerung der GIS-Benutzeroberfláche auf den Internet-Client bedeutet zugleich, daß die elgentliche GIS-Funktionalitüt auf dem Server verbleibt. Die Interaktion des Client mit der GIS-Software (GIS-Server) wird uber den Web-Server vermittelt, indern die im HTTP-Format ubermittelte Anfrage in einen vom GIS interpretierbaren Aufruf obersetzt wird. Unabdingbare Voraussetzung für diese Kopplung ist also das Vorhandensein mindestens einer Schnittstalle sowoh beim GIS-als auch beim Web-Server, Selt der Entwicklung der ersten WobServer am CERN und NSCA hat sich das Common Gateway Interface (CGI) als Standard durchgesetzt, den heute die meisten Web-Server auf unterschiedlichsten Plattformen unterstützen. Über CGI wird vom Web-Server ein eigenständiges Programm gestartet, das in der Lage sein muB. die standardisierte Parameterubergabe auszuwerten und dementsprechend ein Anwendungsprogramm zu starten. Neuere Entwicklungen ermoglichen eine direkte Kommunikation des WebServers mit Anwendungsprogrammen. Hier wird vom Web-Server eine Programmierschnittstelle (API) zur Verfügung gestellt, die die Erweiterung der Server-Software um Funktionen von Anwendungsprogrammen erlaubt. Der wesentliche Unterschied zur CGI-Schnittstelle ist, daß die Verarbeitung der Antrage innerhalb des Web-Server-Prozesses ertolgt, daher schneller abgearbeitet wird, ein hoheres MaB an Integration ermoglicht (machtigere Funktionen), sich aber bel Auftrotan von Fehlern auch weniger stabil verhalt. Fur solche Programmierschnittatelien existieren mehrere herstellerspezifische implementationen. z. B. ISAPI von Microsoft und NSAPI von Netscape, die 2.T. aber auch von Server-Software anderer Hersteller unterstützt werden. Zur Datenbankanbindung bieten einige Web-Server spezielie Schnittstellen, wie ODBC auf der Windows-Plattform und JDBC auf der Basis von Java

\section{2 computing first: internet-fahiges GIS}

Abweichend von der Ausgangsposition in 2.1 kann es unter bestimmten Voraussetzungen sinnvoller sein, eine bestehende proprietäre GIS-Anwendung um Kommunikationswerkzeuge zu erweitern - beispielsweise, wenn bereits eine umfangreiche und an verschiedene Anforderungan angepaßte GIS-Losung besteht, oder wenn nur einfache Netzdienste benotigt worden.

Die Hauptlast der Verarbeitungskapazitat verbleibt hier von vornherein auf 
dem Netzwerk-Client, wobei die Netz. anbindung beispielsweise für den $\mathrm{Zu}$ griff auf zentrale Datenbanken genutzt werden kann. Diese Art der Integration ist eine Plattiorm und Sofiware-spezi. fische Weiterentwicklung, die in der jeweitgen Entwicklungsumgebung unter Nutzung der vom Betriebssystem bereltgestellten Netzwerkdienste ertolgt.

\section{3 components first: Objekt- orientierte GIS-Komponenten}

Die dritte Perspektive zur Realisierung von Internet-GIS besteht in der Nutzung von modernen Entwicklungsumgebungen zur Erstellung von Software-Komponenten, Die plattormunabhängige Programmiersprache Java und ActiveX als Internet-Erweitenung des Windows-Komponentenmodells (COM) ermóglichen es, aut Client-wie aut Server-Seite GIS-Funktionalität bereitzustellen. Die Vision dabei ist, daf in einem objektorientierten Ansatz immer nur die gerade benotigte Funktion als Software-Komponente dynamisch geiaden wird.

Dies bedeutet allerdings, bestehende GIS-Sotware auf der Ebene des Source-Code in eine der beiden Umgebungen zu portieren (re-engeneering) oder die Anwendung vollständig neu zu konzipieren und implementieren. Die auf dem Client ausgeführten Komponenten, Java-Applets und ACtiveX-Controls, unterscheiden sich im wesentlichen in ihrem Verhalten aut dern Client: Wahrend ein Applet als eine (in Java geschriebene und als Byte-Code ubertragene) SottwareKomponente in der vom lokalen System weitgehend abgakoppelten Java Virtual Machine des Web-Browser ausgeführt wird, ist ein ActiveX-Control - derzeit allerdings nur aut Win32Plattiormen - in der Lage, mit anderen lokalen Programmen zu kommunizieren und auch aut tokale Speichermedien zuzugreifen. Die Implementation von GIS-Komponenten aut dem Server erfoigt durch Serviets und serverseltige ActiveX-Controls (DLL's).

Um die Frage, welche der beiden Technologien dabei vorzuziehen seil. wird derzeit auf. z.T. wenig sachlicher Ebene heftig gestritten. Im Zentrum der Debatte stehen die Fragen der Plattformabhảngigkeit der ActiveXTechnologie, der Performance von Java-Anwendungen sowie das Problem der Sicherheitslücken bei der
Ausführung von unbekannter Software aut dem oigenen Fechner. So ist es nicht verwunderlich, daß gerade zwei der tührenden GIS-Hersteller in inren Strateglepapieren (ESRI 1997, INTERGRAPH 1996) kontráre Positionen beziehen.

Eine Entschärfung des Grabenkampfes zwischen Java und ActiveX deutet sich allerdings an, da COM bereits teilweise aut andere Plattormen portiert wurde und auch in beiden Umgebungen Schnittstellen geschaffen worden sind, Komponenten der jeweils anderan "Welt zu integrieren.

\section{Bausteine von GIS-Anwendungen im Internet}

im Anschluß an die Einführung in die Techniken, die heute für die Realisierung von GIS-Anwendungen im Internet zur Verfügung stehen, werden die entstehenden Losungen im folgenden aus Sicht des Anbieters und des Nutzers untersucht. Es wird eine Klassifizierung von WebGIS-Anwendungen nach dem Spektrum threr angebotenen Dienste vorgeschlagen, und die die einzeinen Kategorien kennzeichnenden funktionalen Bausteine werden genauer autgeschlusselt.

\subsection{Kategorien}

In den einzeinen Schritten des Szenarios in der Einleitung entstehen jeweils neve bzw. erweiterte Arten einer GISAnvendung im World-Wide Web. Im folgenden sind fünt entsprechende Kategorien definiert und mit typischen Beispielen - z.T. Prototypen oder Testversionen mit Nutzungseinschrânkungen - belegt Die Benennungen orientieren sich an der von WebGIS.Anbietern gewăhiten Begriffen, an fruheren Ansàtzen zur Klassifizierung von WebGIS (STAHL 1997. Srobl 1997) und am intuitiven Verständnis der Bezeichnungen.

\section{Geodaten-Server}

Geodaten-Server fiefern Geographische informationen zur Offline-Weiterverarbeitung mit lokaler Software auf dem Client-Rechner. Dieser. Dienst setzt sich aus Recherche und der Übermittlung der Geodaten zusammen faccess 10 GIS Data Sets" und "transmission of GIS Data Sets" nach MATUSCHAK 1996 und PENG 1997).

Beim Vermessungsamt Bochum besteht die Möglichkeit, Kartenoroben $\operatorname{der} A L K$ und der Stadtgrundkarte als Vektordaton im EDBS-oder DXF-For- mat abzurufen (Link 1). Auch einige GIS-Hersteller bieten Geodaten in inrem jewelligen Format an, wie 2.B. ESFil mit ArcData Online (Link 2),

\section{Map-Server}

Map-Server ubermitteln Karten zur Online-Visualisierung

Statische Map-Server lietern lediglich vorgefertigte Karten, die typischerweise aus einem GIS in ein Rasterformat exportiert wurden. Der WebGISClient erlaubt dem Benutzer im wesentlichen die Auswahl aus dem vorhandenem Angebot.

Unter diese Kategorie fallen einfache digitale Stadtplane ( $Z B$ die HTMLVersion des Borner Stadiplans, Link 3) aber auch zahireiche .image-Server" wie der Digital Orthophoto Browser des MIT (Link 4).

Beim interaktiven Map-Server kann die Darstellung der Karte, beispielsweise die Farbgebung, durch den $\mathrm{Be}$ nutzer beeinflußt werden. Die Karte wird erst dann auf dem Server erstellt und anschließend zur Visualisierung treigegeben.

Zwel dynamische Map-Server sind das "interactive Mapping Tool" NAIS. Map (Link 5) und das internet GIS-Gateway des JRC (Link 6), das in Richtung eines Online-GIS ausgebaut werden soil.

\section{Online-Auskunftssysteme}

Raumbezogene Online-Auskunftssysteme dienen der Visualisierung vorgefertigter oder interaktiv erstellter Karten, bieten dem Client aber dardbet hinaus thematische und einfache raumbezogene Abfragemoglichke:ten. Dies kann beim Erstellen der Karte oder interaktiv auf Basis einer entspre. chenden Mapping-Komponerite (s,o.) geschehen.

Typische Online-Auskunftssysteme mit Raumbezug sind digitale Umweltinformationssysteme $(z B$, der Digitale Uimweltatlas Berlin, Link 7) und Routenplaner wie die interactive Manhattan Subway Map (Link 8). IRIS (Link 9) ist als System für die visuelle Analyse soziookonomischer Daten konzipiert und ermoglicht das Anzeigen und Abfragen von Sachdaten, verbunden mit einem dynamischen Map-Server.

\section{Onine-GiS}

Online-GIS bieten einen unbeschrankten Zugang zu einem aut einem Server 
bereltgesteliten GIS, Das bedeute: derzeit zugleich, daß nur die auf dem Server vorhandenen Daten genutzt werden können.

Je nachidem, ob das Online-GIS ober eine graphische Benutzerschnittstelle oder über die direkte Eingabe von Befehten (Kommandozeile) angesprochen wird, handelt es sich um eine Lösung für Anwender oder für Experten. Online-GIS unfassen auch solche Systeme, die auf Serverseite mehrere GIS anstevern, wobei dem Benutzer eine einhertiche virtuelle" Oberflache zur Verfugung steht.

Das System GAASSLinks (Link 10) bietel dem Benutzer einige GIS-Funktionen wie Klassifizierung und Putferbildung, die es als Online-GiS charakterisieren. Es beinhaltet zugleich eine interaktivo Mapping-Komponente und einen elementaren Auskunttsdienst auf der gelieferten Rasterkarts (Koordinaten und Objektart der angeklick(en Zelie).

\section{GIS-Funktions-Server}

GIS-Funktions-Server öffnen den entfernten Zugriff auf die Funktionen eines Server-GIS. Die Bearbeitung der vom Client geliejerten Daten erfolg: online oder offline. Analyse-Ergeb. nisse werden I.d.R. ohne Visualisierungsmoglichkeit als Dateien zur lokalen Bearboitung zuruckgeliefert In der Kategorie der GIS-Funktions-Server sind auch solche Dienste vorstellbar. bei denen nicht die Daten auf den Server geschickt, sondern die gewünschte(n) Funktion(en) aus einer Bibliothek des Servers abgerufen und als ausfithrbare Datel oder irn Source. code - au d den Client übertragen werden

Mit "ARCANFO via WWW" (Link 11) besteht ein Grundkorzept for einen Dienst, bel dem Daten und Kommandos an einen Server geschickt werden und das Ergebnis des Aufurags per E-Mail zurückgeliefert wird

Selostverständlich sind die Ubbergange zwischen den hier definierten Klassen fließend. So daß die Einordnung konkreter Anwendungen z.T. problematisch ist. Weiterhin sollte berücksichtigt werden, deß sich eine Reihe von Anwendungen noch in Entwicklung befindet, standig weitere Funktionalitat erhalt und sich damit in Richtung hoherwertiger WebGIS-Dienste (= höhere Kategorie) bewegt.

\subsection{Funktionale Bausteine}

Die Verteilung von bisher monolithischen GIS-Losungen auf mehrere miteinander vernetzte Rechner sowie der Bedeutungsverlust des Verarbeitungsschritts der Dateneingabe bringen es mit sich, dals das im deutschsprachigen Raum vielzitierte EVAP-Konzept mit seiner prozeßorientierten Sichtweise an Galtigkeit verliert. Die heutige Aulfassung von GIS orientiert sich zUnehmend am Benutzer, der weniger am Gesamtsystem interessiert ist. als vielmehr an GIS-Dienstleistungen, die seine spezlifchen Fragestellungen bedienen. Die oben beschriabenen WebGIS-Kategorien sind demnach dadurch charakterisient, welche Dienste der Benutzer "einkauft", unabhângig davon, welche Funktionen im Gesamtsystem auf dem Server ausge. führt werden und wie hochwertig die -Ware" ist, die dem Benutzer am Client geliefert wird.

Ene etwas andere Sichtweise, die die Grundiunktionen Ertassung, Verwaltung, Abfrage, Analyse und Prasentation (s belspielsweise COWEN 1988. MAGULAE/DANGEAMOND 1993) nach ihrer durchschnittlichen Komplexitat anordnet. führt auf die folgenden funktionalen Bausteine von GIS (git fur InternetGIS wie für lokale Systeme):

\begin{tabular}{ll}
\hline Datenerfassung und -verwaltung & - Auswahi und Ubertragung von Geodaten \\
& (als Dateien) \\
& - Formatkonvertierung \\
\hline - Auswahl und Darstellung von Karten & - Kartographische Generalisierung \\
\hline Abfrage & - Abtragen von Sachdaten \\
& - Messen, Zahlen, Berechnen \\
\hline Analyse & - Geostatistische Analyse \\
& - Topologische Abtragen \\
& - Verschnelanzoperatoren wie Pufferbildung \\
\hline
\end{tabular}

Tab. 3.1

\begin{tabular}{l|c|c|c|c}
\hline Kategorie & \multicolumn{3}{|c}{ Dienstegruppen } \\
& $\begin{array}{c}\text { Ertassung und } \\
\text { Verwaltung }\end{array}$ & Prasentation & Ablrage & Analyse \\
\hline Geodaten-Server & $x$ & & & \\
Map-Server & $x$ & $x$ & $x$ & \\
Auskunttssystem & $x$ & $x$ & $x$ & $x$ \\
Online-GIS & $x$ & $x$ & $x$ & $x$ \\
Funktions-Server & & $(x)$ & & \\
\hline
\end{tabular}

Zu den Furiktionsgruppen wurden jeweils - ohne Anspruch auf Vollstandigkeit - konkrete Dienste aufgefunrt, die charakteristisch für heutige bzw wünschenswert für zukänftige WebGIS. Anwendungen sind. Tabelle 3.1. zeigt. welche Dienste-Kombinationen die GIS-Anwendungen der Kategorien aus Abschnitt 3.2. jeweils umfassen.

Aus der Gestall der resultierenden $\mathrm{Ma}$ trix läft sich schilußfoigern, daß die vorgeschlagene Kiassifizierung von Web. GIS-Anwendungen alle denkbaren Dienste-Kombinationen bereits umtaßit. In einer ausführicheren Analyse könnten die gewahlten Dienste-Grup. werden und anhand einzelner konkreter Bausteine eine detailliertere Klassifizierung und Bewertung von WebGISLósungen versucht werden

Die Zusammenstellung in Tab 3.2 soll dagegen einen anderen Aspekt verdeutlichen: Welche Art von Information erhalt der Benutzer bei welcher Art von WebGIS? Sind es Daten, die mit geeigneter lokaler Software weiterverarbeitet werden müssen. ist es eine Karte, evtl. mit angehăngten Attributdaten, also eine informativere" Form von Daten, oder sind es Funktionen. mit denen der Anwender seine eigenen Daten bearbeiten und daraus informationen erzeugen kann? pen jedoch genauer aufgeschlusselt 
Tab. 3.2

\begin{tabular}{l|c|c|c}
\hline Kategorie & \multicolumn{3}{|c}{ Lieferung } \\
& Daten & Graphik & Funktionen \\
\hline Geodaten-Server & $x$ & & \\
Map-Server & & $x$ & \\
Auskunftssystern & & $x$ & \\
Online-GIS & & $x$ & \\
Funiktions-Server & & (x) & $x$ \\
\hline
\end{tabular}

An der Liste der ubermittelten "Ware"Daten, Graphik und/oder Funktionen laßt sich die Leistungstâhigkeit einer WebGIS-Anwendung festmachen. Die aus fremden Daten mehr oder weniger interaktiv erzeugten Karten wird man in vielen Projekten aber $\mathbf{Z . B}$, auch in der althaglichen Anwerndung eines Routenplaners nicht weiterverwenden können. Dagegen hat man bei den bisher noch seltenen Diensten, bei denen man Originaldaten (Vektorformatl) erhall oder gar GIS-Funktionen am Server nutzen oder von dort beziehen kann, ein Höchstmaß an Flexibilităt für die eigene Weiterverarbeitung der Ergebnisse einer WebGIS-Session.

Der getrennte Bezug vori Daten und Funktionen ist bel der Bearbeltung Geographischer Informationen allerdings höchst problernatisch, da Funktionen I. $d R$, for bestimmte Datenmadelle entwickelt wurden. Dies legt die Annahme nahe, daß in Zukunft Geodaten-Server auch GIS-Funktionen, die zu den angebotenen Daten passen. offerieren werden und umgekehrt GIS. Funktions-Server, die bisher keine Daten mitlieferten, zumindest präzise Antorderungen an die zu bearbeitenden Daten stellen. Im objektorientierten Sinne hieBe das, die Daten(objekte) beinhalten gleichzeitig Methoden und wissen damit, wie sie sich zu .verhal. ten" haben. Für GIS im Internet ist dies die logische Folge der allgemeinen GIS-Entwicklung in Richtung einer Modularisierung (vgl, die Aktivitaten des OpenGIS Consortium).

Far die Gliederung von WebGiS bedeutet dies eine Entwicklung in Fichtung komplexerer Dienste und zunehmend weniger Trennscharfe - alle Anwendungen werden zu Online-GIS ausgebaut. An die Stelle der vertikalen Gliederung nach Komplexităt von Diensten wird jedoch eine honzontale Unterscheidung mach Anwendungs. bereichen und somit nach dem Funktionsangebot treten. Das bedeutet, daß Onine-GIS genau dann zusammenge- faßst und móglicherweise miteinander verglichen werden, wenn sie GIS. Funktionen und ggf - Dater für einen bestimmten Anwendungsbereich, Z. B. die Siadtplanung, anbietert.

\section{Fazit}

GIS im Internet unterschedet sich bezuglich des Einsatzgebietes und bezuglich der verwendeten Technologle erheblich vam klassischen Offine-GIS. GIS-Anwendungen im Internet sind derzeit meist auf spezielle Dienste ausgerichtet. Sie wenden sich im algemeinen an eine konkrete Zielgruppe und bieten nur eine sehr spezielle Auswahl an GIS. Funktionalitat. Die Müglichkeiten zur Interaktion und Manipulation durch den Anwender sind noch eingeschrănkt Vor dem Hintergrund einer rasanten Entwicklung dieses Applikationsbereiches muB allerdings betont werden, daß diese Aussagen vermutlich sehr bald uberholt sein werden. Die Vision eines „NetGIS (analog zu "NetPC*), bel dern der nur mit einem Browser ausgestattete Nutzer sowohi Daten, ais auch die durch Benutzeroberflachen unterstützten GIS-Funktionalitaten "nach Bedart" uber das Inter-lintranet bezieht. scheint nicht mehr weit entfernt $z u$ sein.

Aut einem derart dynamischen Markt wird jede neue Implementation sehr bald von der Produktentwicklung eingeholt und Artikel uber jegliche Art von Internet-Technologie sind notwendigerweise veraltet, sobald sie veróftentlicht werden. Aus diesem Grund se auf die WWW-Seiten zu diesem Theme verwiesen (http:/Www giub uni-bonn. de/webgis), auf denen die Autoren bemüht sind, aktuelle Entwicklungen zu berūcksichtigen und weitere Informationen wie eine Vergleichsstudie verschiedener Software-Produkte und eine dokumentierte Bookmark-Sammlung zur Verfügung zu stellen.

Konnte die bisherige Entwicklung als Experimentier-Phase angesehen werden, in der seht viele unterschiedliche Techniken ausprobiert wurden, so werden von nun an immer mehr kommerzielle Anbieter mit neuen Produkten den Markt bedienen. Diese Produkte werden besonders in den folgenden Bereichen in harte Konkurrenz treten:

\section{- Anzahi und Qualitat der} Dienste/Funktionen
- Kompatibilität zu vorhandener Software und zu vorhandenen Datenformaten,

- Benutzertreundichkeit der Systembedienung

- Performance im Netzbetrieb (optmale Verteilung der Recheniast zWschen Client und Sever!

- Server-Lizenzbedingunger (einmallge und laufende Kosten)

Dabei kann man heute wohl schon davon ausgehen, daß sowohl die künftigen Anbieter von Internet-GIS-Lösungen, als auch die potentielen Nutzer nicht mehr mit dem vergleichsweise kleinen Kreis der heutigen GIS-Gemeinde identisch sein wercien. GISorientierte Anwendungen (im Internet oder Offline) werden als Komponenten In großere Sottware-Systeme integriert und damit auch nicht mehr nur von GIS-erfahrenen Nutzern bedient werden. Optimistisch betrachtet konnte dies den Vorteil bringen, daB die GISTechnologie sich nur noch mit GIS. spezifischen Funktioneri bescháftigen muß, da beispielsweise Prásentationstunktionen von einer Graphik-Komponenten des Systerns besser ubernommen werden können Es besteht aber anciererseits ebenso die Gefahr, daß in Folge der Zerlegung Geographischer Informationssysteme in einzelne Funktionskomponenten ein zu kleiner GISKern übrig bleibt und somit die Daseinsberechtigung von GIS-Experten in Frage gestellt werden könnte. Aber dies solite nun nicht in GIS-Kreisen zur Panik oder-noch schlimmer-zu einer Abschottungs-Taktik führen. Im Gegenteli: Die Ottnung von GIS dureh und für das Internet kann auch einen starken Aufschwung fur den gesamten GIS-Bereich bedeuten.

Ist man bereit, sich in die neven GISKomponenten-Techniken einzuarbeiten und sich Fahhigkeiten beispielsweise in der Java oder ActiveX-Programmierung anzueignen, so eroffinet sich ein sehr grcher - theoretisch weltweiter - Markt, um GIS-Dienstleistung beispielsweise in Form von portierbaren GIS-Applikationen über einen Server anzubieten. Eine hoffentlich in naher Zukunft realisierte Standardisierung von Formaten und Schnittstellen (OpenGIS) ist hiertür mit Sicherheit eine entscheidende voraussetzung. Auch wenn künttig GIS-bezogene Funktionalităten in Soltware-Systemen angeboten werden, die bisher kaine GIS-Kompetenz besaßen (Microsoft 
oder Netscape seien hier nur beispielhatt enwähnt), so darf man doch nicht vergessen, dafl sich an den grundlegenden Problemen, die bel der Verarbeitung räumlicher Informationen zu losen sind, nichts ändert, nur weil die Werkzeuge zu ihrer Bearbeitung für einen großßeren Nutzerkreis verfügbar und daher einfacher zu bedienen sein werden. Es wird also auch auf dem Gebiet der GIS-Anwendungen im Internet Fachwissen für Methoden und Modelle raumlicher Informationsverarbeitung gefragt sein. Allerdings wird sich dieses spezielle Einsatzfeld von GIS noch starker an den Bedürnissen des Marktes orientieren und damit noch weniger Impulse durch die theoretische Diskussion erhalten, als es in der bisherige GIS-Entwickung der Fall gewesen is!.

\section{Referenzen}

Cowen, DAvie d, (1988): GIS versus CAD versus DBMS: what are the differences? In: Photogrammetric Engineering and Remote Sensing, 54, S 155t-1565, wiederabgedruckt in: Introductory readings in Geographic information Systems, hrsg. von D.J. Peuquet u. Duane F Marble, London usw, 1990, S. $52-61$

EnNst, WaAfex (1996): Presenting ActiveX, indianapolis: Sams net Publishing.

http://duck.ice cycu edu. $\mathbf{w}$ / - duke/ebook/activex/2/paxim. htm ESRI Environmental Systems Research Institute (Hrsg.)(1997): The Future of GIS on the Internet. An ESRI White Paper. Redlands

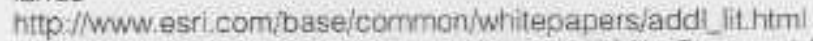
INTERGRAPH Software Solutions (Hrsg.)(1996): Geographic Information Systems (GIS) based on Jupiter Technology. hitp//wwwintergraph com/iss/ipchlibrary/TRENDSIN HTM

MAGuiRE, D. J. U J. Dangemono (1993): .The tunctionality of GIS." In: Geographical Information Systems. Principles and Applications. Vol. 1: Principles, hrsg. von D d. Maguire, M.F. Goodchild und D.W. Rhind New York, S. 319 - 335.

Matuschak Brian 1. (1996): "Commentary: GiS is Being Redefined by Current Computing Trends", in. The Electronic Atlas Newsletter, Vol. 7, Nr. 9

htp / /wwwelectronic-atlas carviean79a.html

Peng, Zhong-Ren (1997): An Assessment of the Development of Internet GIS', in: 1997 ESRI User Conference (Proceedings) Redlands.

http///Wwwesri.combaso/common/userconf/proc97/PRDC97/ TO550/PAP526/P526. HTM

STAHL, ROLAND (1997): GIS und internet Tutorium. Abschnitt GIS - Online.

http /hwww.bonn: netsurt, def Roland. Stahlitutorialinetgision-

line.htm

Sirobe, Josef (1997) .Geo-Datenbasen und Karten im WWW" (Folien zum Vortrag auf der AGIT97 in Salzburg, 2, -4.7.1997). http://wWw.sbg ac, at/geo/agit/agit97/p/str/s/d001.htm

THOAEN, BLI (1995). "Interactive Mapping and GIS Thrive on the Web--, in; GIS World, Oktober 1995, 5, 58 :

\section{Autoren}

JeNs Fizke, Dipl-Geogr, ist wiss, Mitarbeiter an der Universitat Bonn und Projektieiter im Projekt GIS-EXperimental-SErver (EXSE). Adresse: Geographische institute der Universita: Bonn, Meckenheimer Allee 166, 53115 Bonn, Tel, (0228) 73-7508, Fax 5393, e-mail jens.fitzke@uni-bonn.de.

Cuus Rinkn, Dipl.-Systemwiss, ist Doktorand im institut for Angewandte Informationstechnik der GMD St. Augustin. Adresse: GMOIFIT.KI, SchloB Birlinghoven. 53754 Sankt Augustin. Tel. (02241) 14-2401, Fax 2072, 8-mail: Claus, Rinnerggand.de.

DiFK SO-Miot. Dipl-Geogr., ist Doktorand im institut für Angewandte Informationstechnik der GMD St. Augustin, Adresse: GMDJFIT:KI, SchloB Bitlinghoven, 53754 Sankt Augustin, Tel. (02241) 14-2282, Fax.2072, e-mail: dirk.schmidt@gmd.de.

\section{Anhang}

\section{EXSE: GIS-EXperimental-SErver}

(www.giub uni-born dejexse/)

EXSE ist ein Projeit der Abteilung Geographische Intormationssysteme und Fernerkundung der Geographisehen institute der Universitat Bornn in Zusammenarbeit mit der Siamens Nixdorf Intormationssysteme AG und weiteren Projektpartnem. Aniaß des Projektes ist das wachsende interesse von Betreibern Geographischer Informationssysteme, die bisher meist isoliert verwalteten Datanbestânde einem großeren Nutzerkreis uber das internet su erschliefen. In Anbetracht einer zunehmenden Zahl von .WebGIS Soltware" unterschiedilichster Austichtung ist es dringend erforderlich, anhand korkreler Spezifikationen charaiteristische "WebGISAnwendungen" zu implementieren. Diese Spezifikationen werden gemeinsam mit den Projektpartnem, derzeit vor aliem aus der Bffentlichen Verwaitung erarbeitel.

\section{Links}

1. Vermessungs-und Kalasteramt, Bochum: Online-Service. hitto:ilwww bochum, de/vermessungsamtiver $4 . h$ tm

2. ESRI: Arcolats Online hltp:/fwuw esti.com/base/datalonline/browse html

3. Tobias Preußer: Stadtpian Bonn. hup J/cips02.physik.uni-bonn.de/ preusserfplan-ueb. htmi

4. Massachusetts Institute of Technology: MIT Digital Orthophoto Browser htto: $/$ orthomit edu/nscil

5. NAISMap2: Interactive Mapping Tool hitp://ellesmere.com emr.calschochet/issuemapl Home html

6. Joint Research Centre of the European Commissiort: The GIS gateway to the WWW. htto//tawsOB irc.il/gis-gateway/gateway_main html

7. Senatsverwaltung for Stadtantwicklung. Utrweltschutz und Technologie, Berlin. Digitaler Umweltatlas Berlin http:I/Wwwicf delUiSenline/

8. Interactive Manhartan Subway Map http://www transarc.com/ats/transarc com/public/orail/ html/transit/manhattan.htm

9. G. und N. Andrienko. IRIS: a knowledge based system for visual data exploration. http://allanon.gmd de/and/javafiris/

10. SHuse: PEGIS GRASSLinks 3.0: Public Access GIS. http://regis berkeley, edu/grasslinksfindex.htmi

11. S. Lehmkuhler: ARC/INFO via WWW http:/arpplus.raumplanung. unidortmund.de/pinkpant/ pinknet/ (nur Beschreibung)

\section{Glossar}

\begin{tabular}{ll}
\hline CGI (Common Gateway Interface) \\
COM (Component Object Model) \\
FTP (Flle Transter Protocol) \\
HTML (HyperText Markup Language) \\
HITP (HyperText Transfer Protocol) \\
ISAPI (internet Server Appication Programming interface) \\
MIME. (Multipurpose Internet Mail Extensions) \\
NSAPI (Netscape Server Application Programming interfa- \\
Ce) \\
VRML (Virtual Reality Modeling Language)
\end{tabular}

